\title{
PULVERIZED COAL INJECTION FOR HIGH INJECTION RATES*
}

\author{
André Oliveira ${ }^{1}$ \\ Pierre Mahowald ${ }^{2}$ \\ Ben Muller ${ }^{3}$ \\ Klaus-Peter Kinzel ${ }^{4}$ \\ Victor Oliveira ${ }^{5}$
}

\begin{abstract}
The present paper describes state-of-the-art Pulverized Coal Injection technology with focus to Dynamic Distribution guaranteeing highest accuracy on equal distribution and being thus one of the key factors enabling constantly highest injection rates. As an alternative to the construction of a new plant, existing plants may be modernized and upgraded to latest technology. Two recent examples are presented. As a conclusion, two case studies of return on investment are showing that the $\mathrm{PCl}$ technology, both for new facilities as well as for upgrades, is very profitable and mandatory for high blast furnace operation effectiveness.
\end{abstract}

Keywords: Pulverized coal injection; Dynamic distribution; Plant upgrade; TPDS; ROI. Luxembourg.

3 Dipl.-Ing. TH Aachen, Sales Manager, Ironmaking, Paul Wurth S.A., Luxembourg, Luxembourg.

4 Dr.-Ing. INPL, Senior Sales Manager, Ironmaking, Paul Wurth S.A., Luxembourg, Luxembourg.

5 Dipl.-Ing, Project Engineer, Injection Technologies, Ironmaking, Paul Wurth do Brasil LTDA, Belo Horizonte, Brazil. 


\section{INTRODUCTION}

The Paul Wurth Pulverized Coal Injection (PCI) technology is based on experience since 1980, when the first PCl plant has been developed and built. More than 100 $\mathrm{PCl}$ plants with Paul Wurth technology have equipped blast furnaces worldwide ever since. So far Paul Wurth has designed installations with maximum injection rates up to $250 \mathrm{~kg} / \mathrm{t}$ of hot metal whereas conveying distances of up to 1.100 meters between the conveying hoppers and the blast furnace have been realized. The Paul Wurth $\mathrm{PCl}$ system is designed to be able to convey a wide range of coal qualities ranging from lignites (high volatile coal) to anthracites (low volatile coal), thus providing for a beneficial use of metallurgical effects as well as flexibility to fluctuating coal market prices.

The present paper gives a short description of the current state-of-the-art $\mathrm{PCl}$ technology. In addition advantages and examples of possible upgrades are presented. As a conclusion two return on investment case studies show that the $\mathrm{PCl}$ technology remains, even at current market conditions, an interesting investment.

\section{PAUL WURTH STATE OF THE ART PCI TECHNOLOGY [1]}

Recent examples of monthly based injection rates above $240 \mathrm{~kg} / \mathrm{t}_{\mathrm{HM}}$ reached at BF A and B of ArcelorMittal Gent, Belgium, or $200 \mathrm{~kg} / \mathrm{t}_{\mathrm{HM}}$ at BF H of Tata Steel Jamshedpur, India, underline the outstanding performances and very high reliability of Paul Wurth PCl plants. Key aspects of the Paul Wurth PCI technology consist in:

- Dense phase conveying: This type of conveying represents a high ratio of coal versus transport gas which assures low nitrogen consumption, very low velocities in the transport lines and is therefore virtually free of wear. A further considerable advantage of low transport gas rates consists in improved combustion properties once the coal enters the blast furnace raceway.

- Control and Distribution: A reliable pulverized coal flow rate control and an accurate distribution of the global coal flow onto the hot blast tuyeres are key aspects to an efficient replacement of coke in the blast furnace process. As such, Paul Wurth has developed a variety of distribution systems in order to provide tailor-made solutions for each blast furnace size and operation philosophy. Depending on the installed system, an inaccuracy of equal distribution of at maximum $\pm 1 \%$ can be achieved.

o Simplified Static Distribution: Use of a static splitter and same equivalent injection line lengths.

o Regular Static Distribution: Use of a static splitter, same equivalent injection line lengths and subcritical coal tuyeres. 


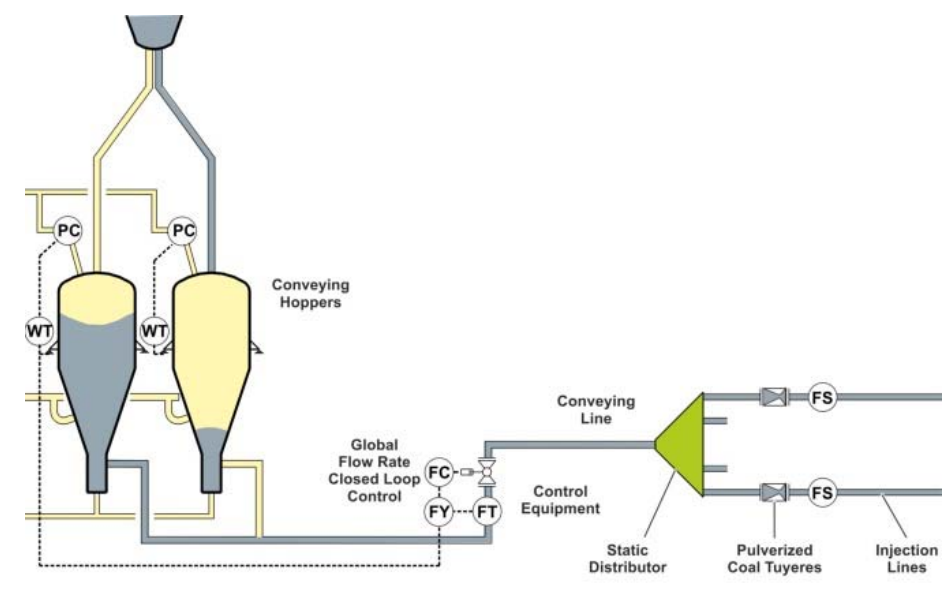

Figure 1. Regular Static Distribution

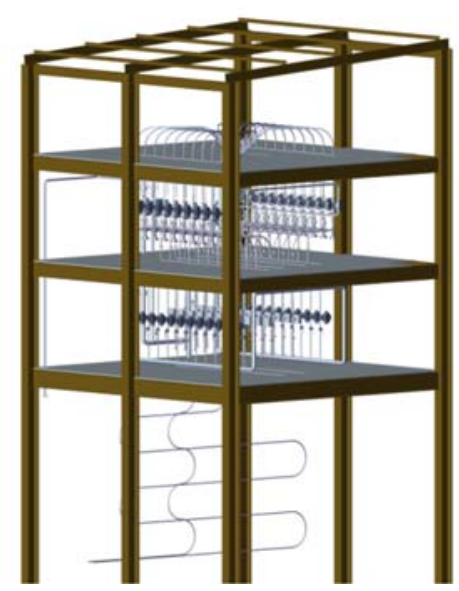

o Dynamic Distribution: Use of a static splitter and closed loop flow control on each injection line. Rough flow resistance balancing only is required, thus no same equivalent pipe line lengths have to be foreseen.

o Distribution with Injection Hopper: Use of an additional pressure vessel located close to the blast furnace, the so-called injection hopper, allowing the best possible preparation of the coal/gas mix combined with closed loop flow control equipment installed in each injection line.
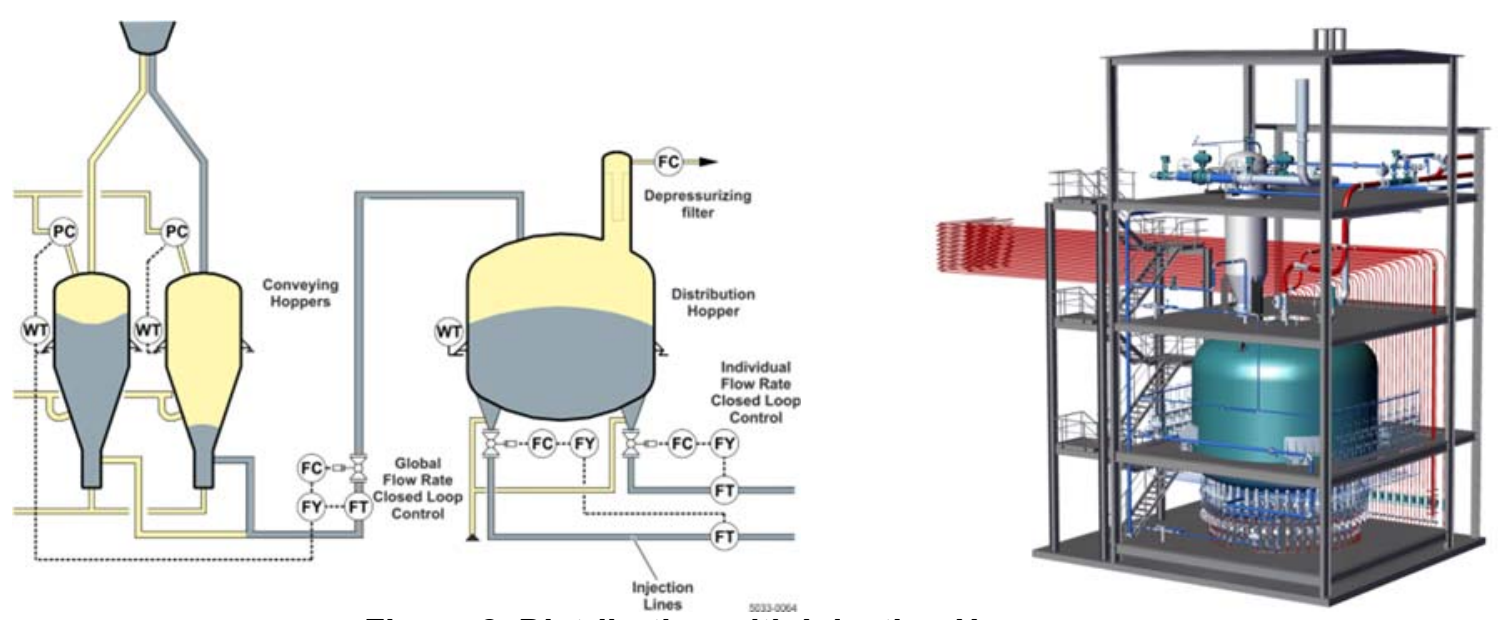

Figure 2. Distribution with Injection Hopper

- Availability and Reliability: A sophisticated automation system enables a maximum of plant availability and flexibility based on the current needs for operation, combined with a very minimum of operator input. The use of equipment provided by highly reputable suppliers guarantees short downtimes for maintenance, a maximum of life expectancy and are therefore the main contributors to the reliable injection of pulverized coal into the blast furnace.

- Key Components: In order to meet the requirements of a high-performance injection facility, Paul Wurth developed a variety of proprietary equipment, such as flow control valves, mixing chambers, splitters and others. These strategic components reflect the experience in coal injection of more than 30 years and are main assets in Paul Wurth's technology portfolio. 

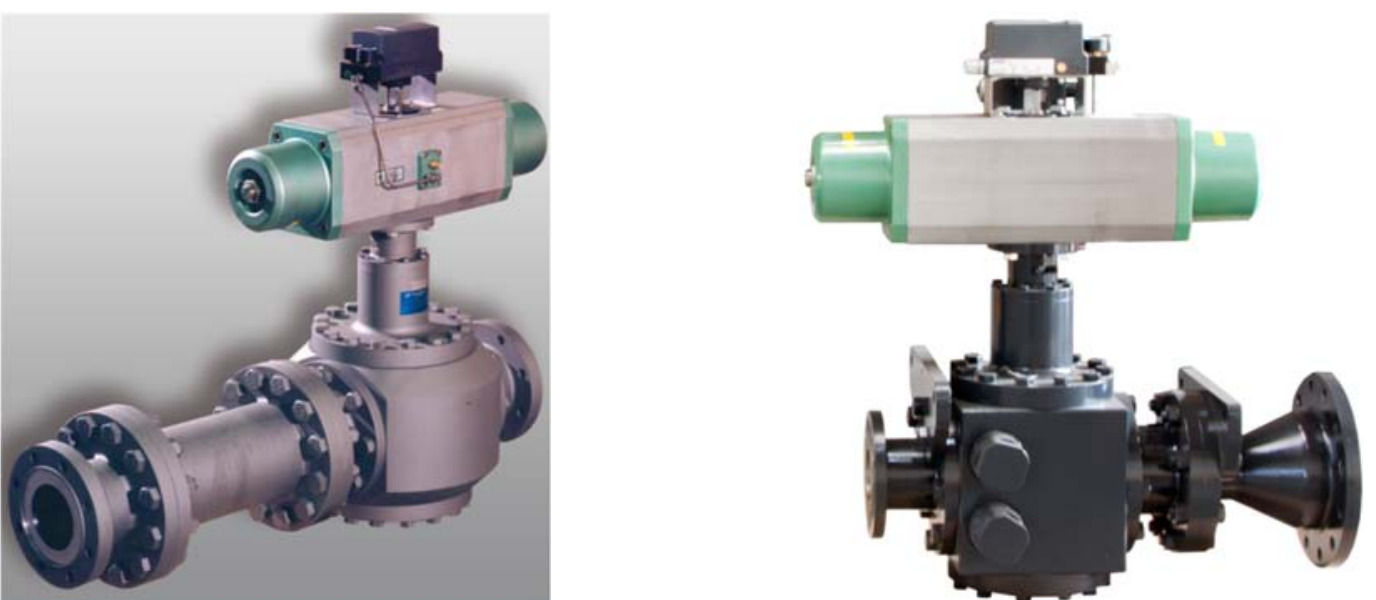

Figure 3. Paul Wurth GRITZKO ${ }^{\circledR}$ coal flow rate control valve and depressurizing flow rate control valve

\section{PAUL WURTH DYNAMIC DISTRIBUTION TECHNOLOGY}

Equal or intentionally non-equal distribution of the total pulverized coal flow to be injected onto all the injection lines in operation and the related downstream hot blast tuyeres is obtained by means of an inline coal flow rate measurement device, a GRITZKO $^{\circledR}$ coal flow rate control valve and a closed loop control installed in each injection line, connecting the distribution device to the related downstream injection lance mounted onto the blow pipe of the related tuyere stock. Advantages of the Paul Wurth Dynamic Distribution technology mainly consist in:

- Increased Distribution Accuracy: A Static Distribution system usually guarantees an inaccuracy of equal distribution to at maximum $\pm 5 \%$, whereas for the Dynamic Distribution technology the inaccuracy of distribution is guaranteed to be at maximum $\pm 2,5 \%$.

- Non-uniform / Non-equal Distribution: The Dynamic Distribution system can also intentionally be set up to a non-uniform and non-equal distribution of coal to the blast furnace tuyeres. This effect can be useful for injection lances in the tap hole area, where a reduced heat input might be of interest. This can be achieved by applying different multiplication factors to the individual injection line set points.

- Continuous Monitoring of Distribution Accuracy: A Dynamic Distribution system permanently monitors the coal flow distribution accuracy. An active closed-loop control balances any deviation and automatically adjusts the position of the GRITZKO $^{\circledR}$ coal flow rate control valve. As distribution is actively closed-loop controlled, any deterioration, e.g. due to coal deposits in injection lines, is balanced and corrected.

- Reduction of Nitrogen Consumption: A slight reduced pressure drop over the GRITZKO ${ }^{\circledR}$ coal flow rate control valve compared to the otherwise installed coal tuyere leads to a slightly reduced nitrogen consumption. The GRITZKO ${ }^{\circledR}$ opening position is always adapted to the actual coal flow rate providing a constant pressure drop, which cannot be realized by means of static coal tuyeres.

- Increased Availability: Compared to static coal tuyeres, the GRITZKO ${ }^{\circledR}$ coal flow rate control valves are also increasing the availability of the installation by strongly reducing the number of plugged lines and by eliminating the need of 
changing the coal nozzles required to achieve a good operation for different injection rate ranges.

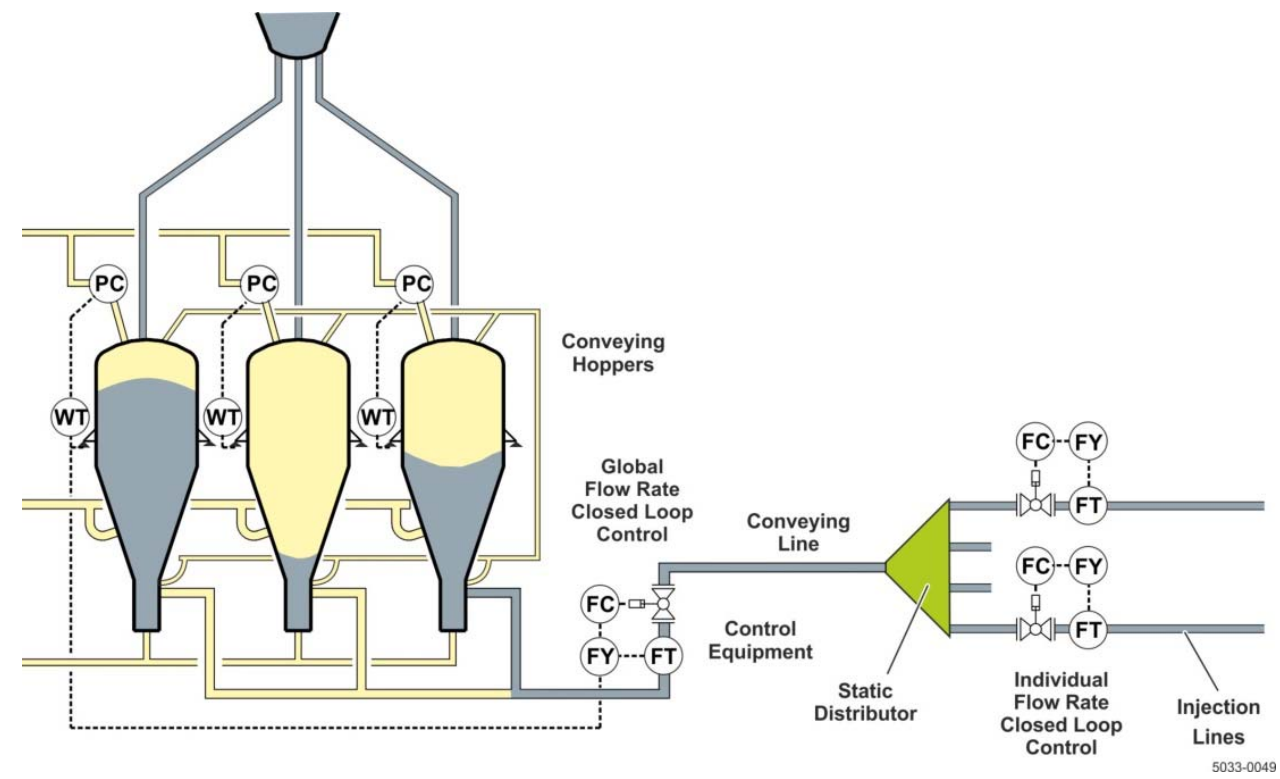

Figure 4. Paul Wurth Dynamic Distribution

\section{IMPROVEMENTS AND UPGRADES OF EXISTING PCI PLANTS}

Paul Wurth's range of products does not only include the design of new facilities but also focuses on improving and upgrading existing plants. The decision for a plant upgrade versus a new-built can be based on several arguments:

- Cost savings: Reusing existing components, equipment and structures can greatly contribute to cost savings.

- Technical focus: Specific addition or replacement of key components and process parameter optimization bear the potential of significant improvement that may prevent unnecessary investments.

- Time constraints: Compared to a new plant construction, upgrades offer a substantial possibility for time savings and subsequently reduce down-times and production losses.

With its vast experience in project management and site support, Paul Wurth is the perfect partner for the execution of feasibility studies and audits that allow for a quick evaluation aiming for the most efficient approach. Short delivery times for engineering and equipment additionally help to shorten project durations and thus speed up the achievement of specified goals. Extensive knowledge in erection and commissioning tasks combined with process support and staff training ensures successful project completion and smooth transition to continuous operation.

\subsection{ArcelorMittal Gent, BF A \& BF B, Belgium [2]}

Arcelor Mittal Gent, Belgium, is the best reference for Paul Wurth for continuous improvement. In collaboration with Paul Wurth, the $\mathrm{PCl}$ technology was initially introduced on BF A and BF B in 1987. Due to frequent upgrades and modernisations of the entire PCl plant, ArcelorMittal Gent is today one of the worldwide leader with injection rates of more than $240 \mathrm{~kg} / \mathrm{t}$ HM on both blast furnaces. 
One of the last modifications on the PCl plant, realized in 2010, was the switch from Static to Dynamic Distribution; this PW technology proved again to be one of the key factors for reaching highest efficiency on blast furnace operation.

In the aim to achieve the shortest possible shutdown time of the $\mathrm{PCl}$ plant, the rack mounted distribution system arrived on site by truck. Beforehand the distribution rack had been completely preassembled and tested in the workshop. The rack contained the static splitter, the GRITZKO ${ }^{\circledR}$ flow rate control valves, the Cabloc flow measurement devices and other process valves. The works on site were mainly reduced to the removal of the former system and the installation and connection of the new rack. The re-commissioning of the $\mathrm{PCl}$ distribution system took 150 hours for BF A and, with only 93 hours, even less for BF B.

The results after the commissioning showed that the availability of the installation was greatly improved. The number of alarms due to blockages in the injection lines was significantly reduced due to the fact that the GRITZKO ${ }^{\circledR}$ coal flow control valves show a lower risk of clogging.

Concerning the equal distribution, the measured average deviation showed values always below $1 \%$, the guaranteed value being $2,5 \%$. This high accuracy on equal distribution leads to a very stable injection. Immediately after the upgrade of the $\mathrm{PCI}$ plants, AM Gent increased the pulverized coal injection rates on both blast furnaces towards the design capacities. Levels up to $234 \mathrm{~kg} / \mathrm{t}_{\mathrm{HM}}$ have been reached on monthly average, without giving in on total RAR.

\subsection{JSW Steel Limited, BF1 at Toranagallu Steel Plant, India}

The pulverized coal injection system of BF1 at JSW Steel Limited, Toranagallu Steel Plant, India, is an example where the nominal injection rate was largely increased from $17,5 \mathrm{t} / \mathrm{h}$ to $50 \mathrm{t} / \mathrm{h}$ with a minimum investment, as most of the existing injection system was reused. The installation was re-commissioned in March 2016.

The existing conveying hoppers were retained and a new intermediate hopper was added. The gain of injection rate was mainly achieved by changing the conveying cycle of the conveying hoppers from continuous to batch wise conveying. By doing so, the conveying hoppers can always convey at maximum flow rate into the newly added intermediate hopper. The intermediate hopper continuously fluidizes and conveys the pulverized coal to the distribution system located at the blast furnace area.

To allow a fast pressurizing of the existing conveying hoppers the fluidizing chambers were modified. Additionally two new pressurizing gas vessels were installed.

The intermediate hopper is equipped with a bag filter to release the excess nitrogen introduced during filling. The pressure inside the intermediate hopper is controlled to be constant in order to achieve a constant injection rate into the blast furnace. 


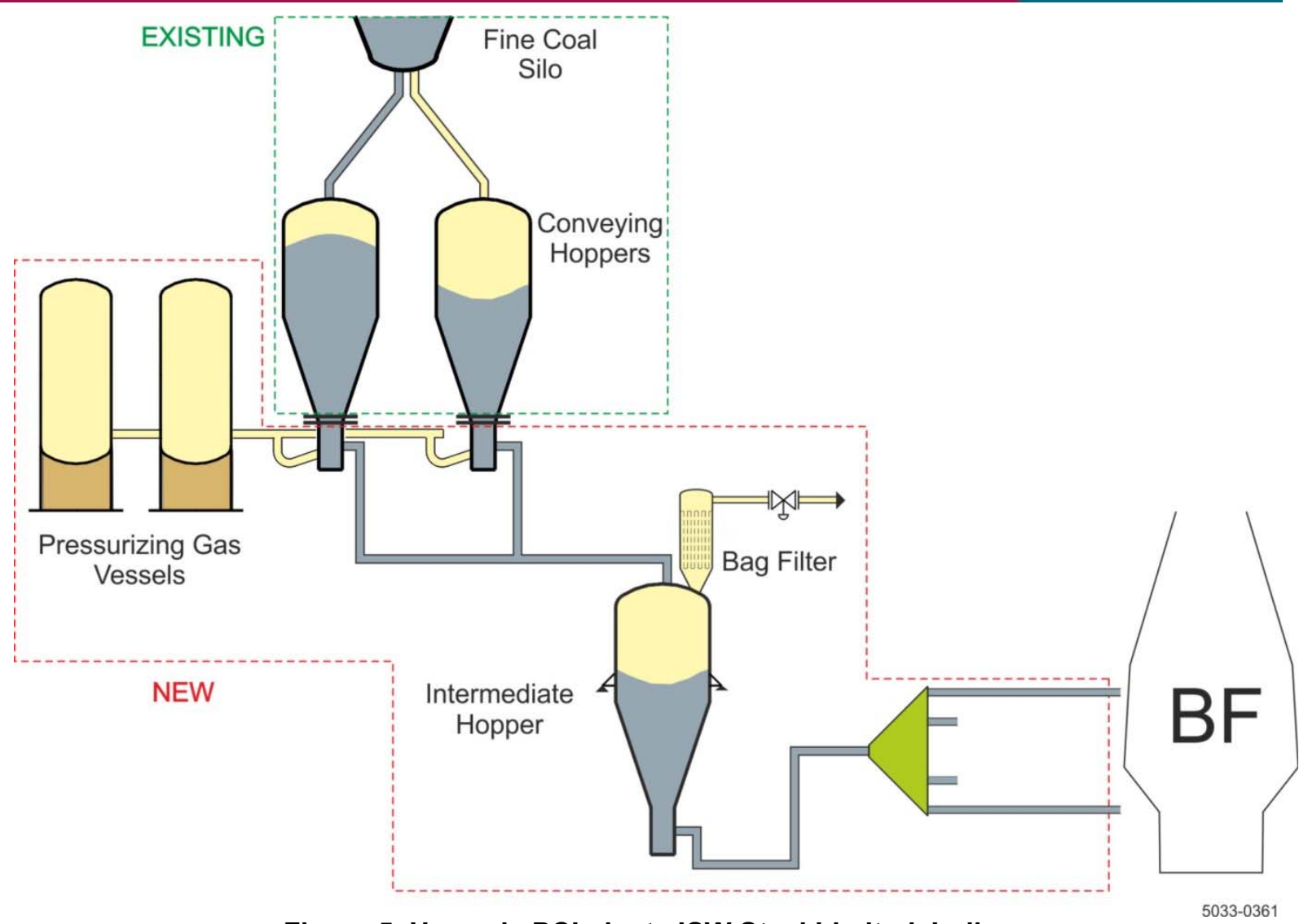

Figure 5. Upgrade PCI plant, JSW Steel Limited, India

While it was not possible to replace the existing conveying hoppers by bigger conveying hoppers, the chosen solution allowed an important gain in capacity of the installation with a reduced investment compared to a complete new installation.

\section{TPDS camera system}

The Tuyere Phenomena Detection System (TPDS) is a proven technology to enhance the safety for personnel and equipment especially beneficial when using $\mathrm{PCl}$. The system consists of a camera embedded in a robust casing installed on the sight glasses on the tuyeres. The images are displayed in real-time on the control system screens of the blast furnace and automatically analyzed by the Paul Wurth TPDS software. The camera is installed rotated at $90^{\circ}$ with a "special" mirror that still enables to look into the hot blast tuyeres. 


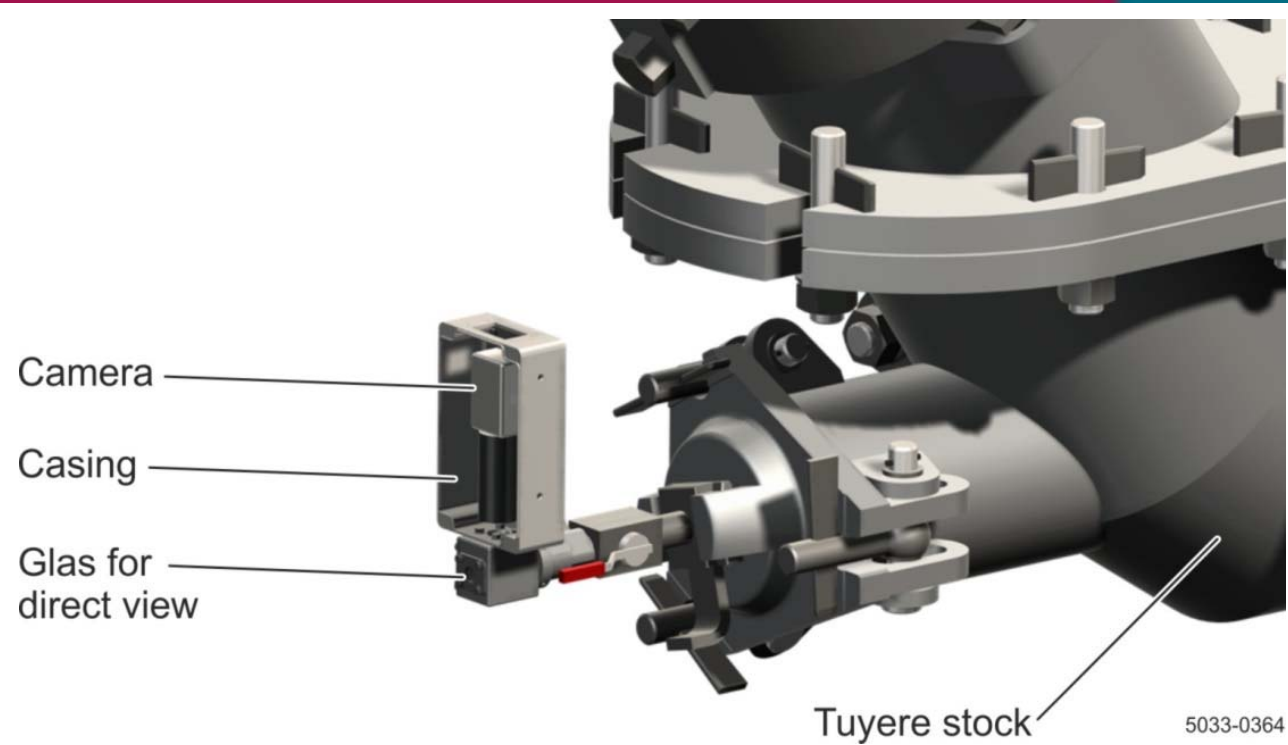

Figure 6. TPDS camera mounted on tuyere stock

The system greatly improves the safety for the personnel as it reduces the manual inspections on the hazardous tuyeres platform. Additionally it brings benefits for the protection of the equipment as the early detection of certain phenomena inside the tuyeres allows reducing the incidental costs.

The system automatically detects different phenomena that can occur inside the tuyeres:

- Blockage of the tuyeres

- Tuyere movement

- Injection lance movement or bending

- Injection lance back burning or breaking

- Injection status

- Ignition state of oxy-coal lances

- Ash or slag deposits at the tuyere tip

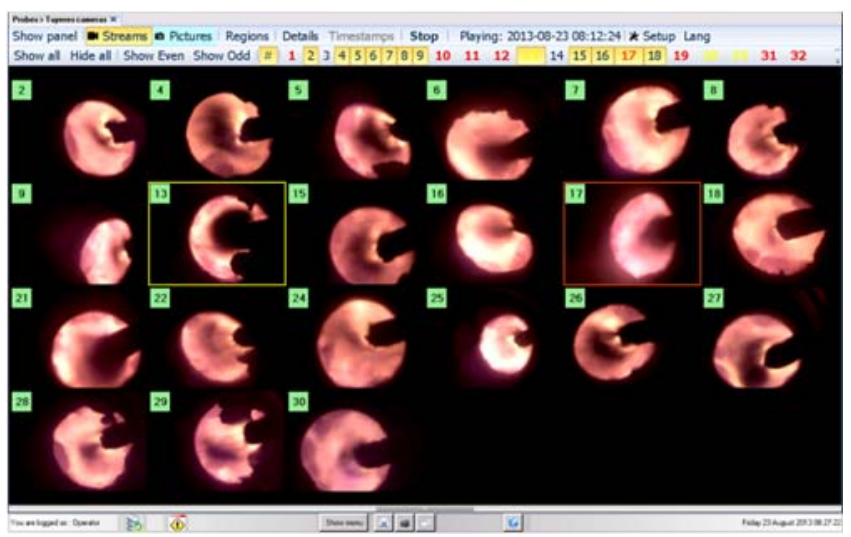

Figure 7. TPDS integrated visualization screen.

\section{RETURN ON INVESTEMENT OF PCI TECHNOLOGY}

During the last 20 years, an enormous productivity increase was achieved at the blast furnace. An important reason for this productivity increase was the possibility to inject reducing agents directly into the lower part of the furnace via the tuyeres. High calorific coal is being more and more utilized today instead of natural gas in order to 
reduce the expensive coke rate. Natural gas injection being limited to roughly $120 \mathrm{~kg} / \mathrm{t}_{\mathrm{HM}}$ and pulverized coal injection allowing higher injection rates of up to $250 \mathrm{~kg} / \mathrm{t}_{\mathrm{HM}}$, co-injection of natural gas and coal may be an economically interesting configuration.

With the today's reduced price difference between coke and pulverized coal, the situation may not seem to be the best time to invest in $\mathrm{PCl}$ technology. However, even under those conditions, the savings due to the coke replacement with cheaper coal will award the investment in short time, as the two case studies below will show. Nevertheless, beside the economies through the coke replacement, there are other positive benefits on the blast furnace.

Lowering of the flame temperature enables an increase in oxygen rates, which, in turn, increases the blast furnace productivity compared to an all-coke operation. Injection of pulverized coal can also assist in maintaining improved furnace stability that results in a more stable consistency of hot iron and slag quality. Fast furnace heat control may lead to a reduced total fuel rate. The higher hydrogen content of coal in comparison to coke leads to an improved blast furnace gas calorific value as well as to a higher reduction efficiency in the blast furnace process.

Last but not least, the improvements by coal injection concerning the environmental aspects are more worth than ever. The reduced coke consumption bears the advantage of lower coke production needs. This results in substantially reduced emissions of $\mathrm{CO}_{2}$ and other waste products of coke making, such as tar and benzol and allows for an active protection of the environment.

\subsection{ROI - Case study for a new medium size $\mathrm{PCl}$ system [3]}

The figure below shows a simplified case study of typical saving potential against typical investment costs for a Grinding And Drying and Pulverized Coal Injection plant, based on the following assumptions:

BF production:

BF production time:

Price delta between coke and coal:

Plant capacity for CAPEX estimation:

Coke replacement ratio for coal:

\section{$4.500 \mathrm{tHm} / \mathrm{d}$}

$350 \mathrm{~d} / \mathrm{y}$ $100 € / t$, incl. OPEX for GAD \& PCl $30 \mathrm{t} / \mathrm{h}\left(160 \mathrm{~kg} / \mathrm{t}_{\mathrm{HM}}\right.$ at $\left.4.500 \mathrm{tHm} / \mathrm{d}\right)$ 0,85 


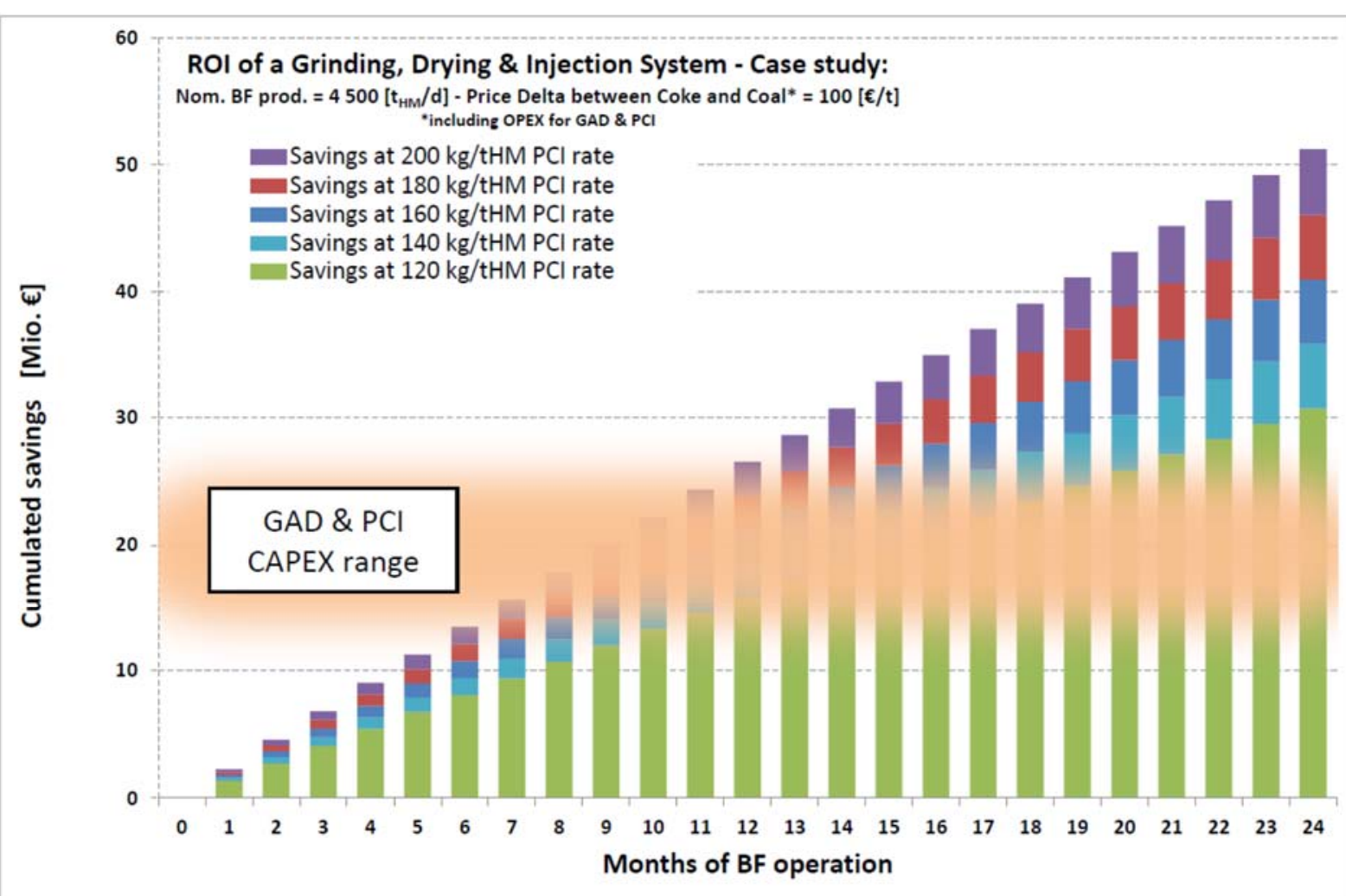

Figure 8. ROI of a $\mathrm{PCl}$ system for a medium size blast furnace

Even when considering a moderate injection rate of $120 \mathrm{~kg} / \mathrm{t}$ нм, a payback time of less than two years can be expected. Such reduced coal injection rates may perfectly be combined with natural gas injection in parallel to coal.

\subsection{ROI - Case study for an upgrade of an existing installation}

The figure below shows the situation for an upgrade from Static Distribution to Dynamic Distribution. For a medium sized blast furnace ROI of 12 months can be achieved with an investment (CAPEX) of about $0,5 \mathrm{M} €$ with an injection rate increased by $6 \mathrm{~kg} / \mathrm{t}$ HM only. For other types of performance upgrades, the ROI can even be shorter. 


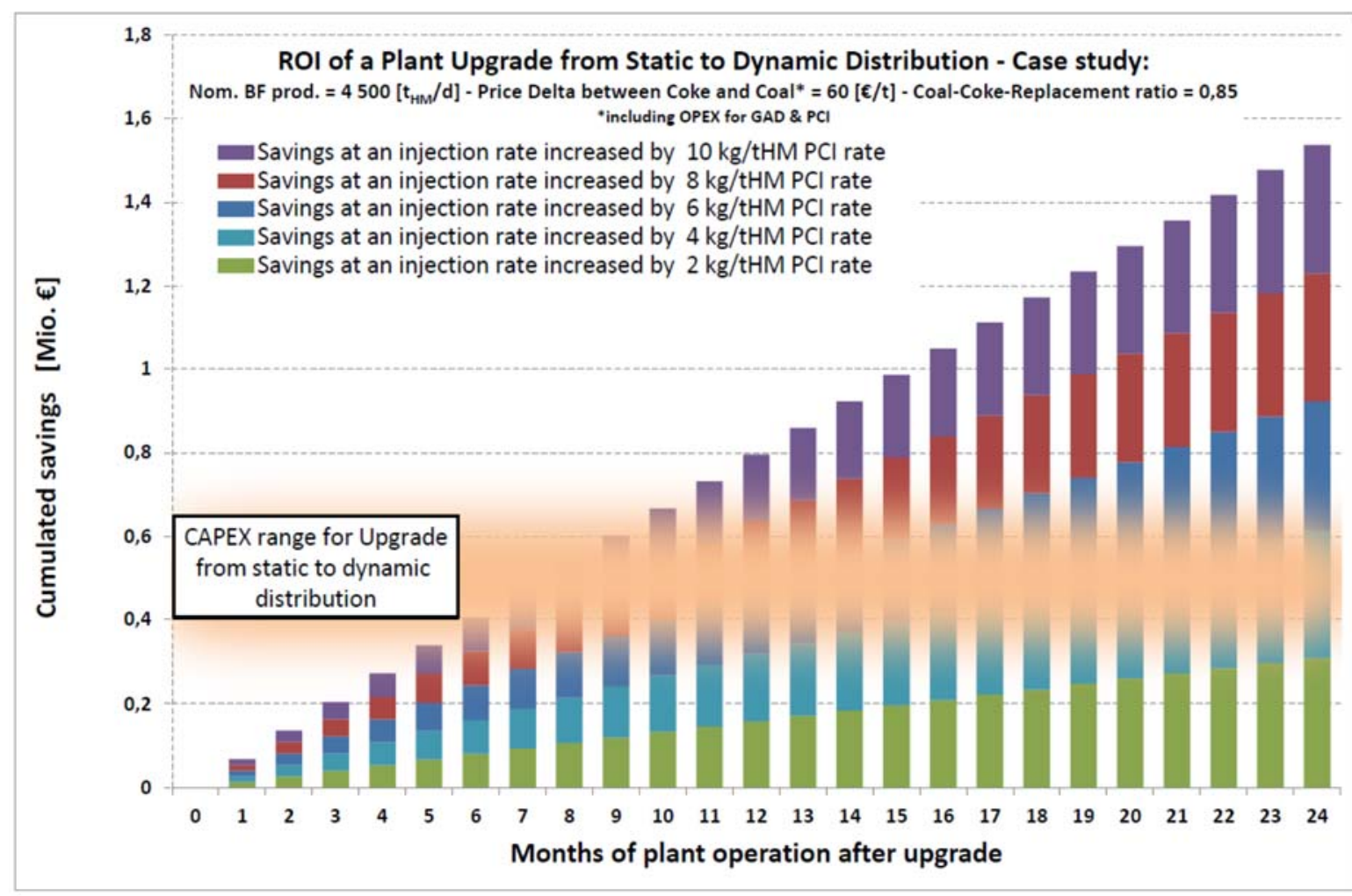

Figure 9. ROI of a PCl upgrade to Paul Wurth Dynamic Distribution

\section{CONCLUSION}

Within the last 30 years the implementation of up-to-date Pulverized Coal Injection technology has proven to be an effective tool to improve blast furnace productivity and efficiency, independent from the blast furnace size. Especially the large potential in coke savings, also if compared with other injectants, make the use of $\mathrm{PCl}$ one of the key factors for substantial cost savings in blast furnace operation worldwide.

With its vast expertise in the furthermost developed technologies of coal processing and injection, such as dense phase conveying, accurate coal flow rate control and distribution accuracy, Paul Wurth is a strong partner for the development of a large variety of projects, starting from green-field installations to upgrading existing plants, with the highest complexity.

\section{REFERENCES}

1 Pierre Mahowald, Ben Muller, Markus Mayer, Emanuele Censi, Salvador Cano. Paul Wurth State-of-the-Art Pulverized Coal Injection Technology. Iron \& Steel Review; 2015

2 Alain Daelman, Pierre Mahowald, Ben Muller. Successful upgrade of pulverized coal injection at ArcelorMittal Gent, Belgium. ABM; 2013

3 David Berdusco, Lionel Hausemer, Paul Tockert, Philipp Bermes, Fabrice Hansen, Steffen Köhler, et al. Investments Facilitating Ironmaking at Reduced Cost. AISTech; 2015. 